\title{
A 3D approach to teaching chemistry
}

Amy A. Sarjeant ${ }^{1}$, Peter A. Wood ${ }^{2}$, Ian J. Bruno ${ }^{2}$, Clare F. Macrae ${ }^{2}$, Helen E. Maynard-Casely ${ }^{3}$, Matthew Towler $^{2}$ ${ }^{1}$ The Cambridge Crystallographic Data Centre, Piscataway, United States, ${ }^{2}$ The Cambridge Crystallographic Data Centre, Cambridge, United Kingdom, ${ }^{3}$ Australian Nuclear Science and Technology Organisation, Kirrawee, Australia E-mail: sarjeant@ccdc.cam.ac.uk

Effective methods of teaching difficult scientific concepts can prove challenging to educators across all academic levels. The inherent wonder and beauty of natural laws are often drowned by tedious textbooks and laborious lectures. For decades, chemistry educators have searched for new and more meaningful ways to communicate science to students who are constantly awash in the instant gratification - and often distraction - of smartphone and other electronic devices. Studies have shown that students who are engaged in active learning, through hands-on experimentation or self-guided inquiry, have better learning rates than their peers whose sole source of knowledge is through textbooks and passive lectures.1 And indeed, in this spirit, the CCDC has sought to augment classroom education by introducing a tactile component through 3D printed molecules.

The Cambridge Structural Database (CSD) contains over 875,000 structures of molecules from simple organic molecules to complex metal-organic frameworks, and everything in between. Structures can be found in the CSD to illustrate many concepts within chemistry curricula, including molecular point group symmetry, intermolecular interactions and a wide variety of conformational concepts like VSEPR theory.

Allowing students to handle these structures, to explore them not only visually but also tactilely, we can stimulate a deeper understanding of learning outcomes such as the connection between structure and function or spectroscopic signatures. Additionally, 3D printed molecules bring crystal structures to life for the visually impaired in a way that no other format can. This presentation will focus on several ways in which 3D crystal structures can enhance chemistry education for students of all levels.

[1] Freeman, S.; Eddy, S. L.; McDonough, M.; Smith, M. K.; Okoroafor, N.; Jordt, H.; Wenderoth, M. P. Active learning increases student performance in science, engineering, and mathematics. Proc. Natl. Acad. Sci. U. S. A. 2014, 111 (23), 8410-8415 DOI: 10.1073/pnas.1319030111.

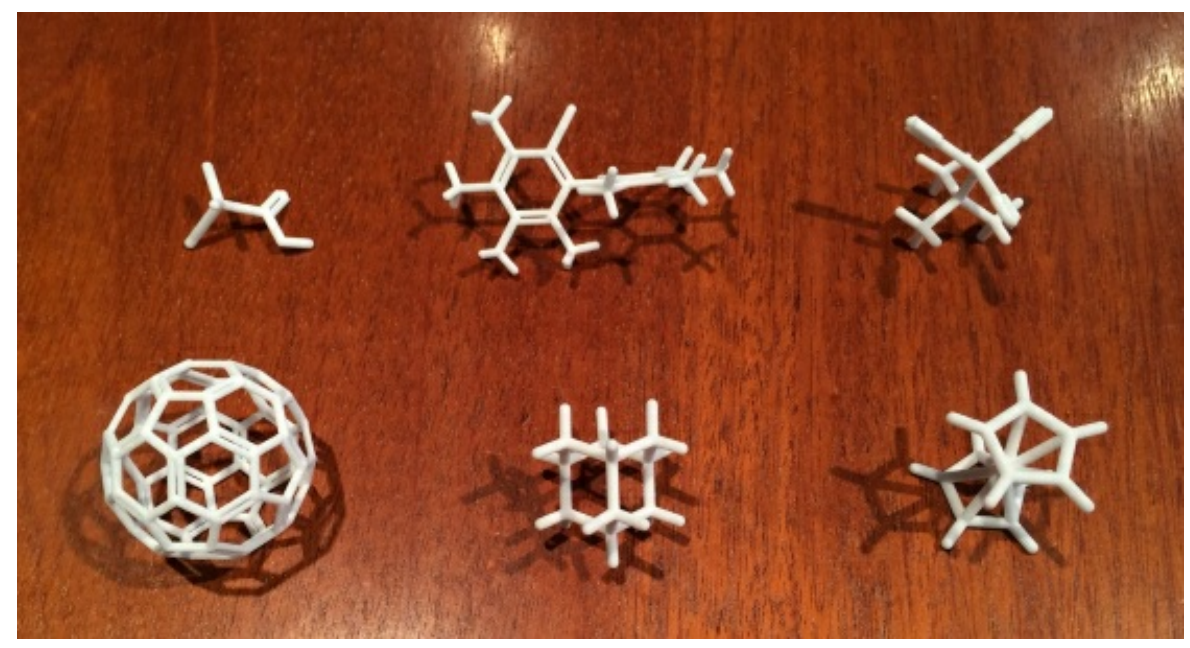

Keywords: education, teaching, 3D printing 